\title{
Improvement of Corona Discharge Model and Its Application on Simulating Corona Discharge in the Presence of Wind
}

\author{
Xiufeng Guo, Qilin Zhang, and Jinbo Zhang \\ Key Laboratory of Meteorological Disaster, Ministry of Education (KLME)/Joint International Research Laboratory of \\ Climate and Environment Change (ILCEC)/Collaborative Innovation Center on Forecast and Evaluation of Meteorological \\ Disaster (CIC-FEMD)/Key Laboratory for Aerosol-Cloud-Precipitation of China Meteorological Administration, \\ Nanjing University of Information Science \& Technology, Nanjing, China \\ Correspondence should be addressed to Qilin Zhang; zhangqilin71@163.com
}

Received 4 May 2017; Revised 7 July 2017; Accepted 29 August 2017; Published 16 November 2017

Academic Editor: Babak Shotorban

Copyright (c) 2017 Xiufeng Guo et al. This is an open access article distributed under the Creative Commons Attribution License, which permits unrestricted use, distribution, and reproduction in any medium, provided the original work is properly cited.

\begin{abstract}
To evaluate the effect of wind on corona discharges occurring on the tip of a grounded rod during a negative charging process of thundercloud, a two-dimensional numerical model has been improved by considering the wind velocity as a driving force for the movement of the corona charges. It was found that not only wind speed but also wind direction have a significant effect on the distribution of corona charges, the local electric field around the rod, and the corona current. (1) Under the same wind speed, a larger horizontal wind can result in less accumulation of corona charges, a larger electric field, and a larger corona current. However, when the speed is less than $5 \mathrm{~m} \mathrm{~s}^{-1}$, the effect of wind direction on the corona current was weak. (2) Under the same wind direction, a larger wind speed can cause a larger corona current. However, when the horizontal wind component is smaller than the vertical, the larger wind speed would cause a smaller electric field. Thus, it is necessary to take the effects of the wind direction into consideration, rather than to consider its speed only, when studying the corona discharge and its effects on the upward leader.
\end{abstract}

\section{Introduction}

As noted by Macgorman et al. [1], the corona discharge, which is often referred to as a "point discharge," usually occurs in a variety of situations and locations, whenever the ambient electric field exceeds the corona onset threshold. The impact of the space charge generated from the corona discharge on the electric field at ground level or on the lightning attachment process has been an important issue studied by many scholars for a long time [2-6]. The corona discharge, as the initial discharge stage when the lightning flash attaches to ground objects, such as the buildings and transmission lines, may produce sufficient space charge which has screening effect on the local electric field. Consequently, the corona discharge may inhibit the inception of the streamer discharge and delay the inception and propagation of the upward leaders [7-10] and further affect the lightning attachment process.

Many factors, such as the aerosol particle density, the air humidity, rain, and wind, can affect the corona discharge.
The effect of wind on corona discharge has been studied both experimentally and numerically by various authors [1115]. D'Alessandro [12] measured the relationship between the peak value of corona current and the speed of wind, and Du et al. [14] have measured and analyzed the variations in corona charge intensity and corona current under different wind conditions. Bazelyan et al. [13] used a one-dimensional approximation model presented by Aleksandrov et al. [16] to evaluate the effect of wind on the glow corona of a grounded electrode under thunderstorms and found that a moderate wind speed of $15 \mathrm{~m} \mathrm{~s}^{-1}$ increased the corona current peak value by more than $150 \%$. However, they assumed that the wind blew radially from the rod tip, which is of no practical significance. By using a 2D symmetrical model, Becerra [17] argued that the simulated results of Bazelyan et al. [13] overestimated the influence of the corona discharge. Zou et al. [15] have developed a 2D time-dependent model and found that the corona current increases almost linearly with the horizontal wind speed, but the effect of vertical wind on the corona current was not considered. Furthermore, in 
the observation of upward lightning in winter thunderstorm in Japan, self-initiated upward lightning always starts from the stationary tower under a larger wind speed [8], because the removal of space charge by wind may facilitate the initiation of an upward leader. During a thunderstorm, the wind may blow in any direction, that is, may have horizontal and vertical components. But, in the above researches, only the wind speed has been considered while direction has not. On the other hand, because of the presence of wind, the distribution of corona charges would be asymmetric. Therefore, a numerical model in the Cartesian coordinate system, which can simulate an asymmetric structure, is necessary. The models above, no matter in $1 \mathrm{D}$ or in $1.5 \mathrm{D}(2 \mathrm{D}$ in symmetrical), can hardly simulate the wind as a vector.

In this paper, we will improve the two-dimensional timedependent numerical model in the Cartesian coordinate system developed by Guo and Zhang [18], by considering the wind velocity as a dynamic term in the model. Then we will study in detail the effects of wind velocity on the corona discharge initiated from a grounded rod tip in the electric field produced by a thunderstorm.

\section{Formulation of the Two-Dimensional Time-Dependent Numerical Model}

2.1. Introduction of the Time-Dependent Numerical Model of Corona Drift. Although in most of the current numerical simulations $[9,10,13,16,17]$, the corona discharge on the grounded objects is seen as symmetric. In practice, corona discharges on a grounded object during a thunderstorm can hardly avoid the presence of the wind. When the wind is present, the drift of the corona ions is usually asymmetric. Thus, when studying the effect of wind on corona discharge, the axisymmetric mode is inapplicable and the model in the Cartesian coordinate system is necessary. Although the effect of wind on corona discharge on a grounded object is a threedimensional problem, it is assumed in this study that the wind in the whole $3 \mathrm{D}$ space is the same and the chosen vertical plane for the study is parallel to the wind vector. Then the $3 \mathrm{D}$ problem can be simplified as a $2 \mathrm{D}$ problem. Based on the two-dimensional numerical model in the Cartesian coordinate system developed by Guo and Zhang [18], which was achieved to compute the corona discharge on two heightunequal objects without wind; we improved this model to study the corona discharge on a single rod with wind present.

Under the negatively charged thundercloud (see Figure 1), the background electric field at ground level is upward and the corona discharge is positive, and the positive corona ions are produced from the tip of an object. From Guo and Zhang [18], two assumptions were used to simplify the corona discharge process. First, because we are interested in the timedependent production and evolution of corona ions in larger space domain of several hundreds of meters, the thickness of the ionization layer within several of millimeters near the corona discharge surface is ignored. According to Kaptzov's assumption, the local electric field near a corona-producing surface is equal to onset corona field $\left(\mathbf{E}_{\text {cor }}\right)$ [16], whose assumption is valid for a nonstationary corona when the background electric field changes slowly $[19,20]$. Secondly, it

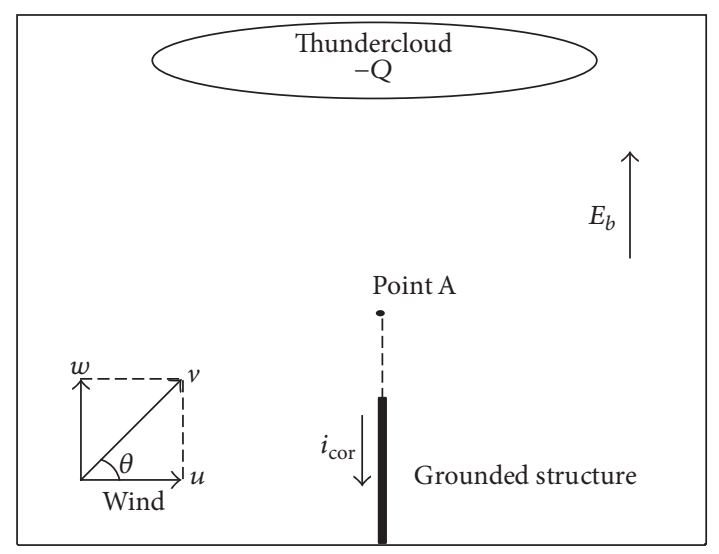

Figure 1: Configuration of the two-dimensional (2D) timedependent numerical model.

is assumed that only the positive corona ions are considered and move away from the corona-producing surface, while all the negative ions in the ionization layer are instantly absorbed by the anode.

According to the above assumptions, the set of continuity equations [18] for small positive ions $n_{+}$, large positive aerosol ions $N_{+}$, and aerosol neutrals $N_{a}$ in 2D Cartesian coordinates are given by

$$
\begin{aligned}
& \frac{\partial n_{+}}{\partial t}=D \cdot \nabla^{2} n_{+}-\nabla \cdot\left(n_{+} \cdot \mu_{n_{+}} \cdot \mathbf{E}\right)-k_{n N} \cdot n_{+} \cdot N_{a} \\
& \frac{\partial N_{+}}{\partial t}=D \cdot \nabla^{2} N_{+}-\nabla \cdot\left(N_{+} \cdot \mu_{N_{+}} \cdot \mathbf{E}\right)+k_{n N} \cdot n_{+} \cdot N_{a} \\
& \frac{\partial N_{a}}{\partial t}=D \cdot \nabla^{2} N_{a}-k_{n N} \cdot n_{+} \cdot N_{a},
\end{aligned}
$$

where $t$ is the time, $\mu_{n_{+}}$and $\mu_{N_{+}}$are the mobility for small positive ions and large positive aerosol ions, respectively, $k_{n N}$ is the attachment coefficient of small ions to aerosol particles, $D$ is the diffusion coefficient, and $\mathbf{E}$ is the electric field. According to the literatures $[2,17,18]$, the values of $\mu_{n_{+}}$, $\mu_{N_{+}}, k_{n N}$, and $D$ are $1.5 \times 10^{-4} \mathrm{~m}^{2} \mathrm{~s}^{-1} \mathrm{v}^{-1}, 1.5 \times 10^{-6} \mathrm{~m}^{2} \mathrm{~s}^{-1} \mathrm{v}^{-1}$, $2.9 \times 10^{-12} \mathrm{~m}^{3} \mathrm{~s}^{-1}$, and $1 \mathrm{~m}^{2} \mathrm{~s}^{-1}$, respectively. Because the size of aerosols is not considered, the value of $D$ is chosen as an average value [2]. Equations (1)-(3) are coupled with Poisson's equation given by

$$
\nabla^{2} \varphi=-\frac{e\left(n_{+}+N_{+}\right)}{\varepsilon_{0}},
$$

where $\varepsilon_{0}$ is the permittivity of air, $e$ is the electron charge, and $\varphi$ is the electric potential.

In the computed method, the second-order upwind difference scheme $[19,20]$ is used to discretize the convection terms (the second term on the right side of the (1) and (2)) from (1) to (3) in order to eliminate numerical oscillation, and the central difference scheme is used to discretize the diffusion terms (the first term on the right side of (1)-(3)). Poisson's equation is solved by the five-point finite difference 
method (FDM), and the successive overrelaxation (SOR) method is used for solving linear systems. Once the potential distribution is obtained, the electric field is calculated as follows:

$$
\mathbf{E}=-\nabla \varphi
$$

\subsection{The Improved Model for Analyzing the \\ Effect of Wind on Corona Discharge}

2.2.1. Introduction of the Improved Model. In order to analyze the effect of wind on the production and drift of positive corona ions generated from the tip of grounded structure, both of the blowing direction and the wind speed have been considered. As shown in Figure 1, the wind direction is described by the angle $\theta$ (degree).

Wind as another driving force plays an important role in the movement of the corona charges and the aerosol particles in the corona discharge. Thus, the continuity equations (1)-(3) in Guo and Zhang [18] should be rewritten as follows:

$$
\begin{aligned}
\frac{\partial n_{+}}{\partial t}= & D \cdot \nabla^{2} n_{+}-\nabla \cdot\left[n_{+} \cdot\left(\mu_{n_{+}} \cdot \mathbf{E}+\mathbf{v}\right)\right]-k_{n N} \cdot n_{+} \\
& \cdot N_{a} \\
\frac{\partial N_{+}}{\partial t}= & D \cdot \nabla^{2} N_{+}-\nabla \cdot\left[N_{+} \cdot\left(\mu_{N_{+}} \cdot \mathbf{E}+\mathbf{v}\right)\right]+k_{n N} \cdot n_{+} \\
& \cdot N_{a} \\
\frac{\partial N_{a}}{\partial t}= & D \cdot \nabla^{2} N_{a}-\nabla \cdot\left(N_{a} \cdot \mathbf{v}\right)-k_{n N} \cdot n_{+} \cdot N_{a},
\end{aligned}
$$

where $\mathbf{v}$ is the wind velocity, which is a vector, and others are as the same as what has been defined in (1)-(3).

It should be stated that the wind considered in this study is mainly focused on the horizontal air flow and the updraft under a thunderstorm while the downdraft is not studied, because the area at ground level underneath a thundercloud with downdraft present is usually accompanied by precipitation while updraft is often without it [21]. The precipitation is also a factor that can affect the distribution of corona space charges. The effect of downdraft on corona discharge cannot be investigated without the presence of precipitation. So the downdraft wind condition is distinguished with the other two wind conditions and its effects will not be investigated in this study but will be studied in the future. Thus the effect of wind on corona discharge is only considered in updraft and horizontal air flow for excluding the influence of precipitation.

It is known that, in the ionization layer, the magnitude of electric field is about several $\mathrm{MV} \mathrm{m}^{-1}$ and the velocity of ions is at least in several hundred meters per second. The velocity of wind at the ground level, which is usually about $10 \mathrm{~m} \mathrm{~s}^{-1}$ during thunderstorms, is much smaller than the velocity of ions drifted in the ionization layer. Thus, the effect of wind on the drift of ions in the ionization layer is ignored and that in the ions diffusion area is mostly investigated in this study. The diffusion area defined in this model is all the area out of the rod surface. And because the thickness of the ionization layer of the corona discharge is ignored, the hyperfine spatial resolution of the computational grids is not a necessity when simulating the ions drift in the diffusion area of a corona discharge in a large space domain. And there is another reason that we choose a coarse spatial resolution. One purpose of this study is to call attention to the effect of wind direction on the corona discharge and to show the numerical model used to simulate this case should be in the Cartesian coordinate system. And we mostly focused on the qualitative analysis of the effect of wind on corona discharge not on the quantitative analysis. Although the gird size may have great influence on the value of the corona properties, the relationship between corona properties and wind may not change too much in the studies with different grid size.

2.2.2. Model Set-Up. The space domain of the analysis is defined from the ground level to the upper boundary with the height of $500 \mathrm{~m}$ while the width in the horizontal direction is $200 \mathrm{~m}$. The structure and its tip are simplified as a lightning rod placed in the middle part of the space domain on the ground. The height of the rod is $60 \mathrm{~m}$ with a radius of $0.5 \mathrm{~m}$. Thus, the spatial resolution of the computational grids is uniform in the domain and is set to be $1 \mathrm{~m} \times 1 \mathrm{~m}$. The temporal resolution matches the spatial resolution and is set to be $0.01 \mathrm{~s}$.

The boundary conditions for the electrostatic calculation are defined as follows. A potential applied to the upper boundary is equal to $E_{b} \times H$, where $E_{b}$ is the time-dependent background electric field (shown in Figure 1) and $H$ is the height of the upper boundary above the ground level. During the formation of a negatively charged thundercloud, the background electric field close to the ground level slowly increases from the fair weather field (about $100 \mathrm{~V} \mathrm{~m}^{-1}$ ) up to a few tens of kilovolts per meter [2] in about 10-30 s [10]. After this, the thundercloud electric field is approximately constant for several minutes until a lightning discharge occurs. Thus, according to Becerra et al. $[17,18]$, it is assumed that the $E_{b}$ increases linearly up to $20 \mathrm{kV} \mathrm{m}^{-1}$ in $10 \mathrm{~s}$, and then remains constant. The right and left vertical boundaries are defined as electric insulators, and the other boundaries including the rods surfaces are set to be the ground potential.

Also, for solving the continuous equations (6), the ground is considered as a zero flux boundary and other boundaries are set to be convection edges. The rod surface where the local electric field equal to or larger than the onset corona field $E_{\text {cor }}$ is defined as the concentration boundaries, generating a corona discharge according to Kaptzov's assumption, and the number of small positive corona ions $\left(n_{+}\right)$created from lightning rod surfaces is estimated by this assumption. In order to evaluate the number of small positive ions created from lightning rod surface according to Kaptzov's assumption, the electric field at the rod surface should maintain its electric field within the range $E_{\text {cor }} \pm 1 \%$ in this model. The details of Kaptzov's assumption can be referred to Guo and Zhang [18]. The initial conditions are as follows: the number density of aerosol neutrals $N_{a}$ is assumed to be $10^{11} \mathrm{~m}^{-3}$ in the air [18], and the initial values of $n_{+}$and $N_{+}$are assumed to be zero.

It should be noted that the corona onset electric field $E_{\text {cor }}$, commonly estimated with Peek's law, is an extremely 


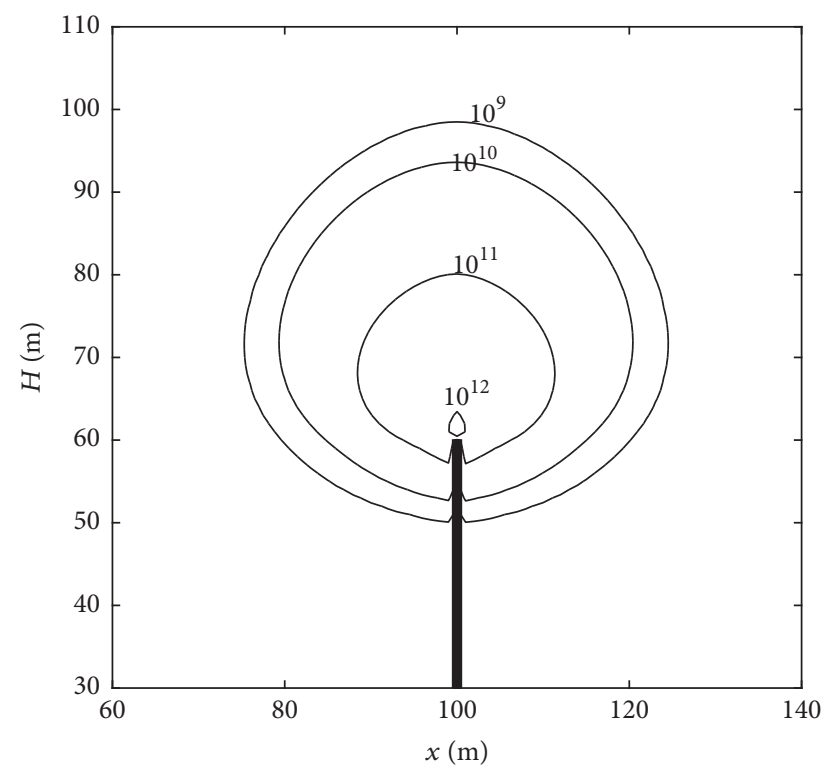

(a)

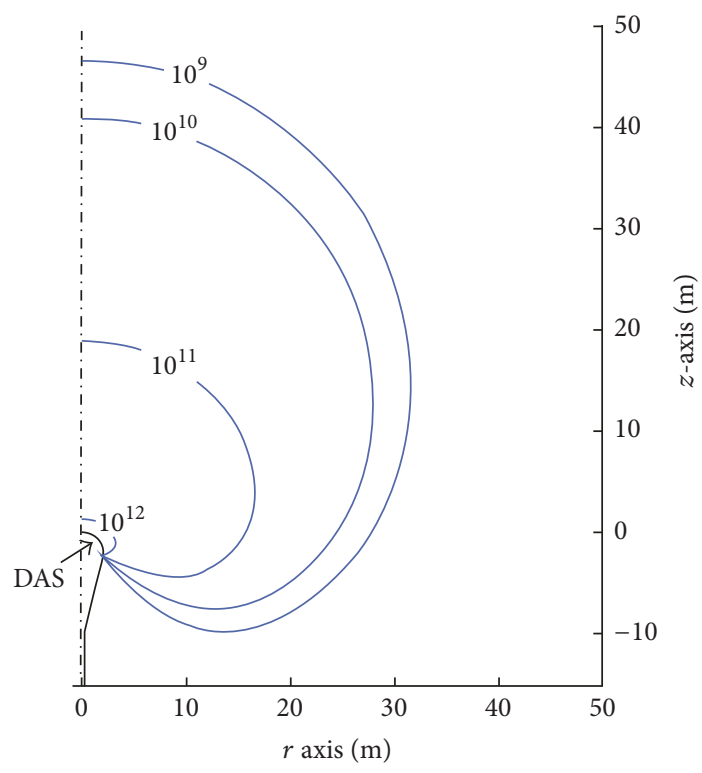

(b)

FIGURE 2: Contours of the number density of small positive corona ions (per cubic meter) produced by the corona from a $60 \mathrm{~m}$ tall grounded rod tip computed with the 2D model in the Cartesian coordinate system (a) and contour plots adapted from Figure 14 in Becerra [17] (b). The result corresponds to the background electric field reaches at $20 \mathrm{kV} \mathrm{m}^{-1}$ produced by thunderstorm.

important critical condition. It can be found from Peek's law that the $E_{\text {cor }}$ is a constant for a lightning rod with a fixed dimension in a certain relative air density. However, in the computed method, the computed electric field on the rod surface is greatly dependent on the chosen spatial resolution; different spatial resolutions can lead to different computed results of the electrostatic field [22]. Based on the relationship of the electric field at the rod surface with the spatial resolutions shown by Tan et al. [22] and the onset electric field $E_{\text {cor }}$ set in Guo and Zhang [18], $E_{\text {cor }}$ under the standard condition with the uniform spatial resolution of $1 \mathrm{~m} \times 1 \mathrm{~m}$ is $1.107 \mathrm{kV} \mathrm{cm}^{-1}$. Therefore, in our model the computed field value of $1.107 \mathrm{kV} \mathrm{cm}^{-1}$ is chosen as the ionization threshold field.

2.3. Validation of Our Numerical Model. Figure 2 displays our computed contour plot of the density of small positive ions without considering the effect of wind. From comparison, it is found that our simulated results are very similar to that presented by Becerra [17] (see their Figure 14). However, in our modeling, our computed time lasts $5.7 \mathrm{~s}$, while their computed time is $10 \mathrm{~s}$; therefore, the diffusion region of small positive ions as shown in Figure 2 in our model is a little smaller than theirs. Figure 3 further shows the computed time variation of the corona current, which is very similar to the variation tendency of the background field assumed in our model. It is because the fact that the corona discharges have a close relationship with the increment of the ambient electric field. The more increment of field value results in a more corona discharge. However, the corona discharge will decrease when the background field keeps a constant. The total ambient electric field around the rod tip can be divided into three parts, which are the background electric field from the thunderstorm, the screening electric field from the corona space charges, and the two electric fields intensified by the rod. For a rod with a fixed dimension, the variation of the electric field is dependent on the change of the background electric field and the screening electric field. When the background electric field is kept as a constant, the variation of electric field around the rod tip is only caused by the drift of the corona charges in the space around it. But this electric field variation caused by corona charges movement is weaker than that caused by background electric field, and then the generation of new corona charges begins decreasing. It makes corona current decrease. In other words, the physical process of our model is reasonable and can be used to study the corona discharge generated from the rod under the different wind condition.

\section{Analysis of the Simulated Results}

3.1. The Effects of Wind on the Diffusion Region of Corona Charges. When the computed field value on the surface of the rod tip exceeds the ionization threshold field $E_{\text {cor }}$, the corona discharge occurs, and different directions and speed values of wind can result in different temporal and spatial distributions of corona ions. As shown in Figure 4, when the background electric field reaches its maximum value at $t=5.7 \mathrm{~s}$, the spatial distributions of the positive corona ions are different in different wind conditions. Both the wind direction and speed have considerable effects on the spatial distribution of the corona ions. For the horizontally blowing wind (see Figure 4(b)), the positive corona density above the rod tip is much less than that of other cases, and space charges are mostly cumulated at the rod's leeside. For the same wind speed value, the more horizontal wind component can result 


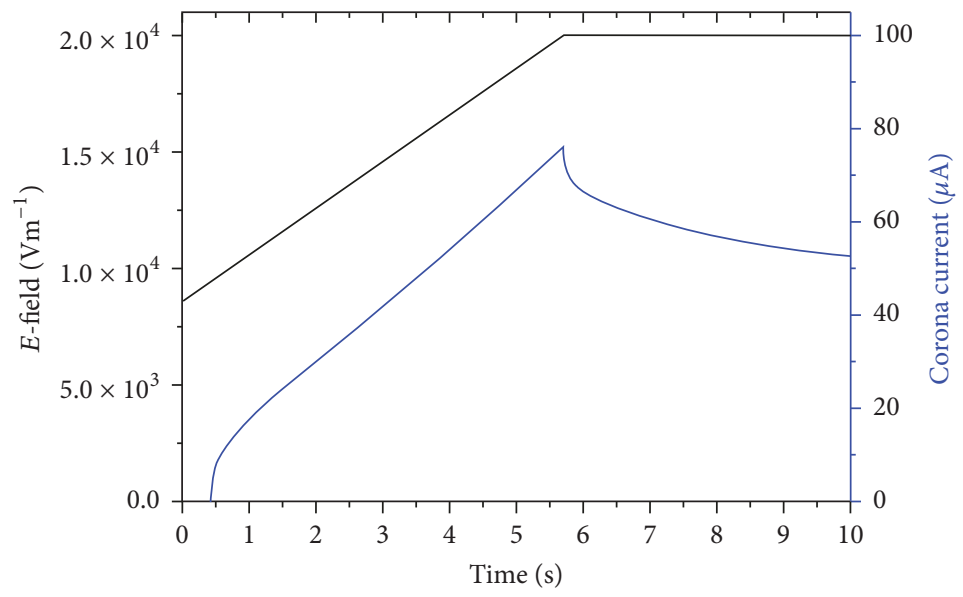

Figure 3: Computed time variation of the corona current (blue curve) of a $60 \mathrm{~m}$ tall grounded rod tip under the background thundercloud electric field (black curve).

in an easier corona discharge, because more corona ions are blown farther away from the rod. Therefore, under the negatively charged thunderstorm, it is easier for the upward leader to propagate when the more horizontal wind component blows. And, in the meantime, the electric field will be reduced on the leeside of the rod because of the shielding effect of corona space charges. This may support the observation of [8] that the thunderstorm electric fields at which upward lightning discharges occur are usually very low.

\subsection{The Effects of Wind on the Local Electric Fields above the} Rod Tip. From Section 3.1, different wind conditions result in a different space distribution of corona ions density above the rod tip, which further affects the local electric fields around the rod tip. The space charge is distributed in the space volume and no unique position can define where the shielding effect is acting. But in order to study the effects of wind on the local electric fields above the rod tip, we just select point $A$ as an example which is located at $4 \mathrm{~m}$ above the rod tip. Figure 5 shows the effect of wind on local electric field at point $A$ as shown in Figure 1.

Figures 5(a) and 5(b) show the effect of wind direction and speed on the temporal evolution of the electric field at point $\mathrm{A}$, respectively. To view as a whole, it can be found that the local electric field at point A increases linearly and then keeps a constant value. From Figure 5(a), it is found that the larger the horizontal wind speed, the more the local field value, because the larger horizontal wind speed results in a more obvious movement of corona ions which further reduce the screening effect on the local field of point A. For example, the peak value of the electric field at $t=5.7 \mathrm{~s}$ increases from 65 to $85 \mathrm{kV} \mathrm{m}^{-1}$ as the horizontal wind speed increases from 0 to $15 \mathrm{~m} \mathrm{~s}^{-1}$. From Figure 5(b), for the wind speed of $15 \mathrm{~m} \mathrm{~s}^{-1}$, when the direction of the wind changes from horizontal to vertical (e.g., the degree $\theta$ increases from $0^{\circ}$ to $90^{\circ}$ ), the peak value of the electric field at $t=5.7 \mathrm{~s}$ decreases from 85 to $56 \mathrm{kV} \mathrm{m}^{-1}$.

Furthermore, Figures 6(a) and 6(b) present the peak electric field at point $\mathrm{A}$ as a function of the wind speed and its direction, respectively, at $t=5.7 \mathrm{~s}$ at the moment when the thunderstorm field reaches a maximum. From Figure 6(a), the effect of the horizontal wind on the field value at point $A$ is different from the vertical wind. For the horizontal wind (see the line for $\theta=0$ ), the larger wind speed results in a larger field value, while the larger wind speed results in a lesser field value for the vertical wind (see the line for $\theta=90^{\circ}$ ), because the former causes the corona ions to deviate from the rod tip (see Figure 4), while the latter causes the corona ions to move up and result in a more screening effect on the point $\mathrm{A}$ near rod surface. The field peak value at the point $\mathrm{A}$ increases linearly with the wind speed for the direction angle less than $45^{\circ}$ but decreases linearly with the wind speed for $\theta$ greater than $45^{\circ}$.

In other words, the local field value at point A is closely dependent on the space distribution of positive corona ions. When the horizontal wind component is much larger than the vertical wind component, the larger wind speed results in a more field value, and when the horizontal wind component is much less than the vertical wind component, the larger wind speed may result in a less field value.

3.3. The Effects of Wind on the Corona Current at the Rod Tip. The corona current is an important physical parameter, because it is closely related to the variation of the background electric fields. Several authors even detected the thunderstorm background field by measuring the corona current occurring at the rod tip under thunderstorm process [23, 24]. However, the corona current is dependent on many factors including the air humidity, rain, and wind $[13,25,26]$. In the following section, we will study the effect of wind on the corona current.

Similar to the field characteristic at point A, as shown in Figure $7(\mathrm{a})$, the corona current at the rod tip linearly increases with the background thunderstorm electric field, and the corona current decreases after the background field reaches a maximum at $t=5.7 \mathrm{~s}$. Because after keeping the background electric field as a constant, the increment of the total electric field around the rod tip is only dependent on the 


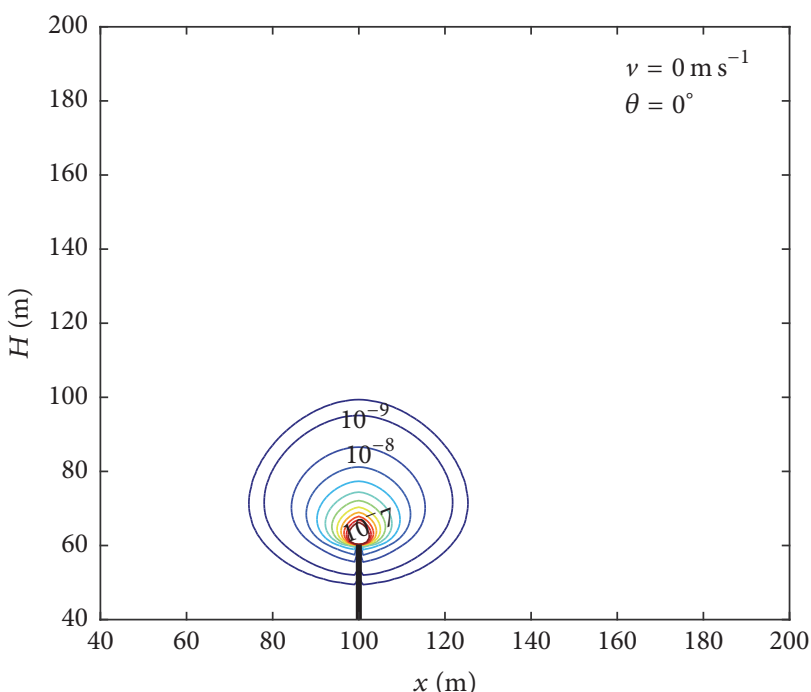

(a)

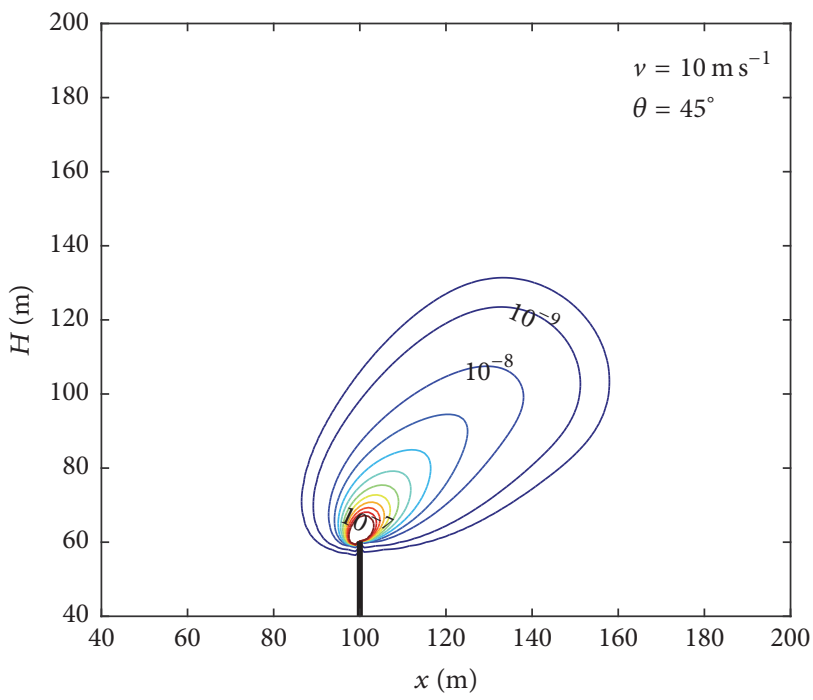

(c)

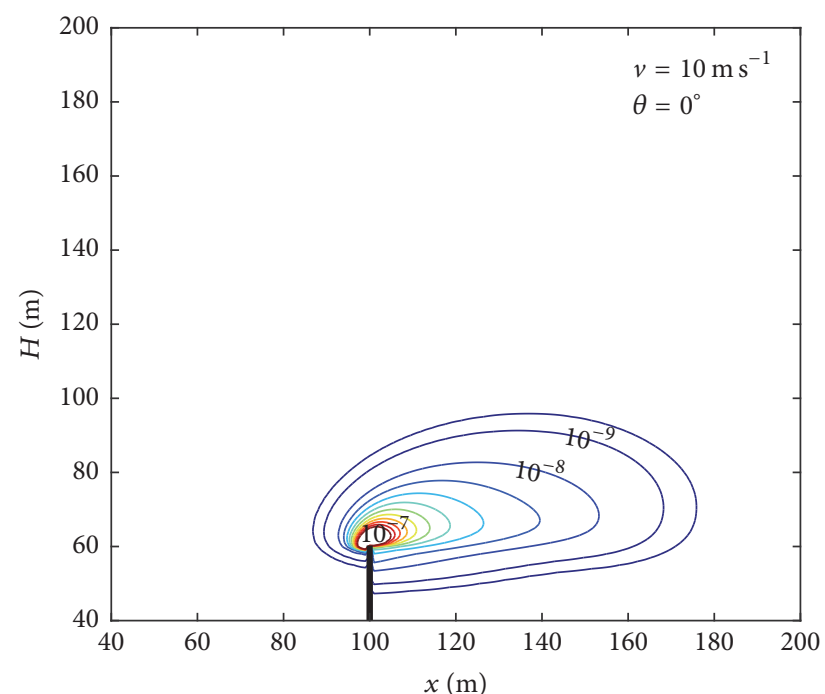

(b)

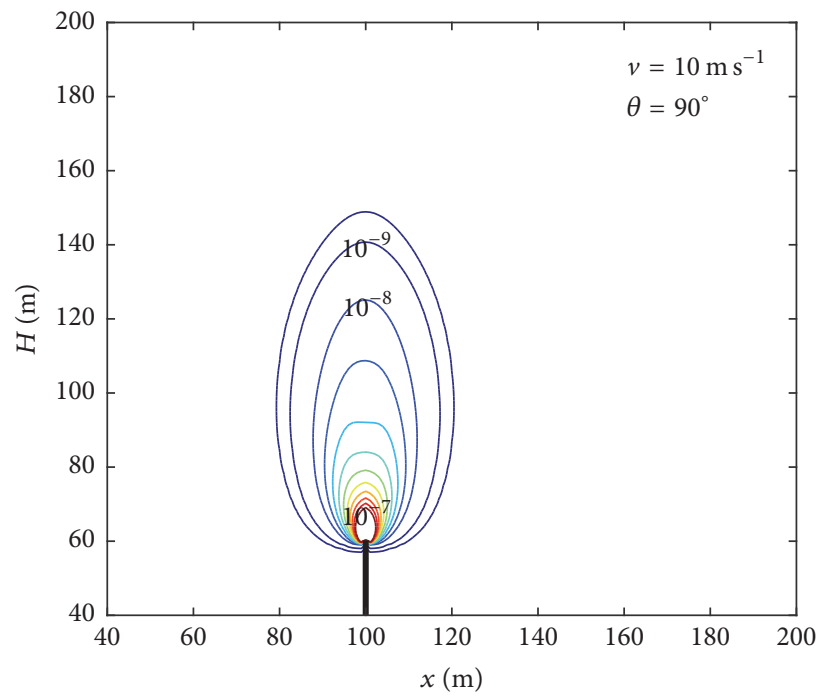

(d)

FIGURE 4: Contours of the corona charge density $\left(\mathrm{C} \mathrm{m}^{-1}\right)$ above the rod tip of $60 \mathrm{~m}$ for wind speeds $v=0$ (a) and $v=10 \mathrm{~m} \mathrm{~s}^{-1}$ with different directions (b, c, and d), and the simulated results correspond to the background electric field of $20 \mathrm{kV} \mathrm{m}^{-1}$ produced by thunderstorm.

decrease of the screening electric field caused by the corona space charge drifted away from the rod tip. But this electric field increment is weaker than that caused by the background electric field, and then the generation of new corona charges begins decreasing. It makes corona current decrease. For the horizontally blowing wind, the peak value of the corona current increases from 70 to $145 \mu \mathrm{A}$ as the wind speed increases from 0 to $15 \mathrm{~m} \mathrm{~s}^{-1}$. From Figure 7(b), for the same wind speed of $15 \mathrm{~m} \mathrm{~s}^{-1}$, when the wind direction changes from horizontal to vertical (e.g., the degree $\theta$ increases from $0^{\circ}$ to $90^{\circ}$ ), the peak corona current decreases from 145 to $100 \mu \mathrm{A}$.

Figure 8 further shows the peak corona current as a function of the wind speed (a) and the direction (b). It is shown that both of the more horizontal wind components or larger wind speed will result in a larger corona current. Because they can cause the positive corona ions to draft away from the rod tip and make a more decrement of the screening electric field. The corona current has a linear variation with the wind speed. And this relationship also has been found in the experimental study by D'Alessandro [12]. The simulation result is close to that of the experimental study, which further validates the effect of this numerical model. Furthermore, this study shows the variation of the corona current with the wind direction. When the speed is fixed, the more vertical components will result in a smaller corona current. However, when the wind speed is less than $5 \mathrm{~m} \mathrm{~s}^{-1}$, the effect of wind direction on the corona current is not obvious.

\section{Conclusions and Discussions}

The impact of the space charge generated from the corona discharge on the electric field at ground level or on the 


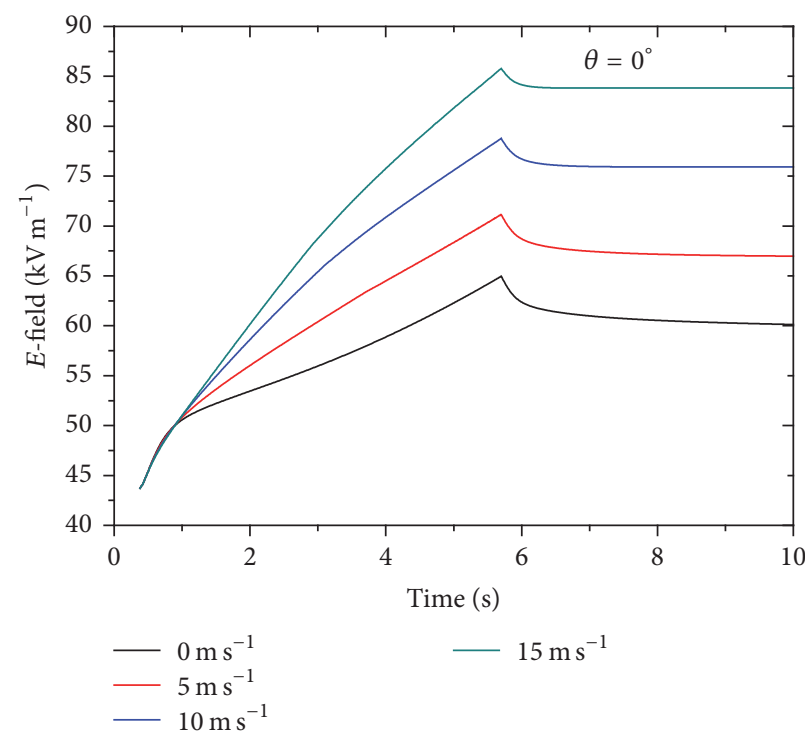

(a)

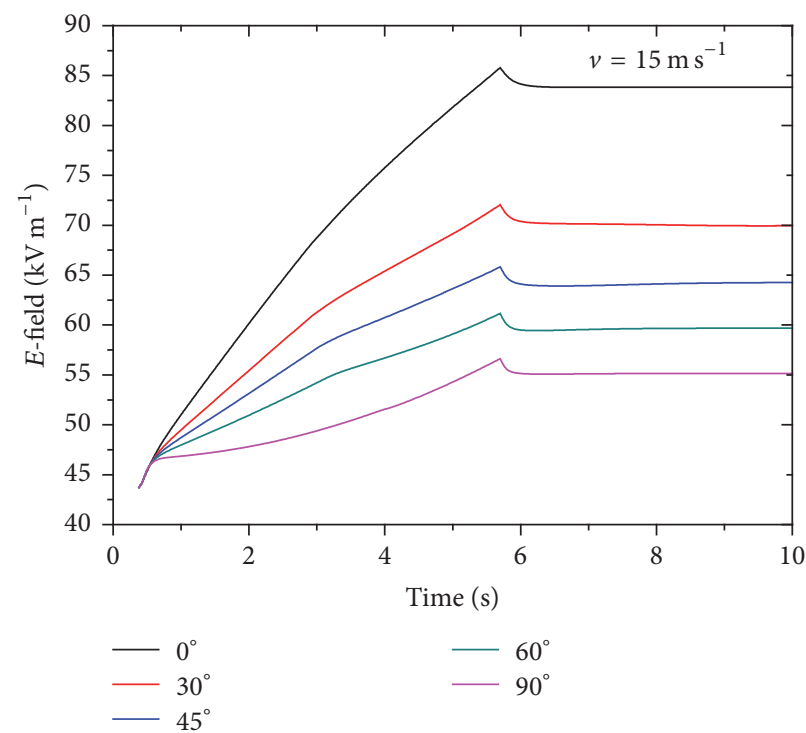

(b)

FIgURE 5: The time-evolution of the field value at the point A for different wind speeds with the same direction (a) and for different wind directions with the same speed value (b).

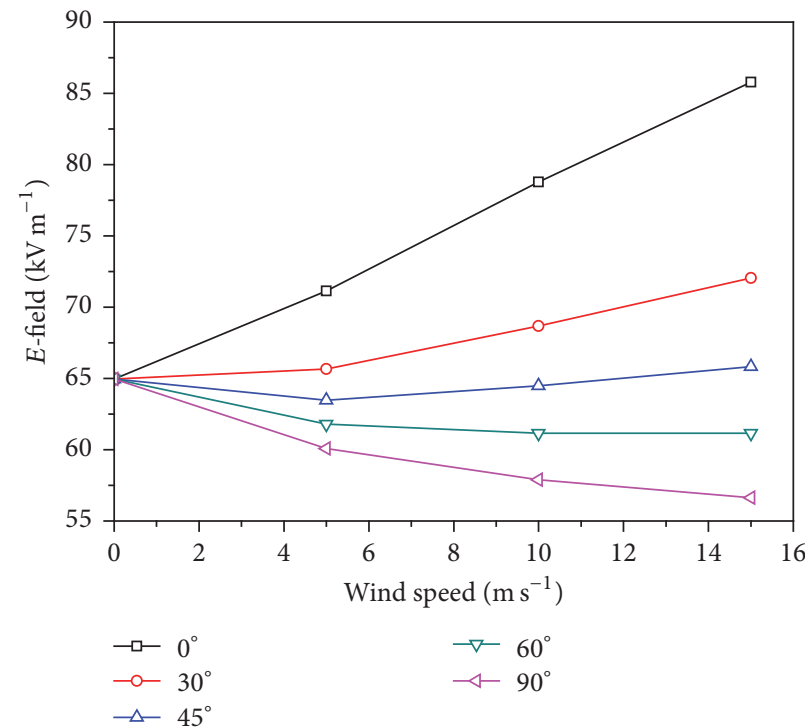

(a)

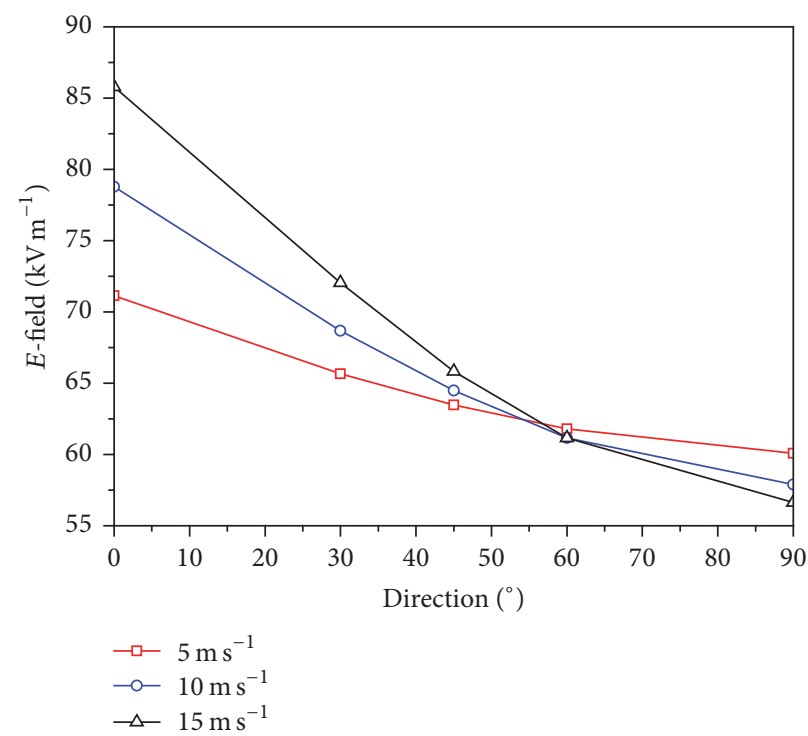

(b)

FIgUre 6: The peak electric field at the point A as a function of the wind speed (a) and its direction (b).

lightning attachment process has been an important issue of concern to many scholars for a long time. Although the effect of wind speed on corona discharge has been studied both experimentally and numerically by various authors, the studies are rarely found to consider the wind as a vector and to discuss whether or not the wind direction can affect the corona discharge and to evaluate the degree of its effect on the corona parameters. Therefore, in this paper, we have studied in detail the effects of wind on the corona discharge initiated from a grounded rod tip in the electric field produced by a thunderstorm by developing a two-dimensional timedependent numerical model.
It was found that both of the wind speed and direction have significant effects on the draft regions of the corona charges, the local electric field above the rod tip, and corona current. Firstly, under the same conditions, the more horizontal wind component can result in an easier corona discharge because more corona ions are blown farther away from the rod. Under the negatively charged thunderstorm, we speculated that the upward leader or the upward lightning can propagate easier with a larger horizontal wind component. Secondly, the local field value at point A at $4 \mathrm{~m}$ above the rod tip of $60 \mathrm{~m}$ is closely dependent on the space distribution of positive corona ions. When the horizontal 


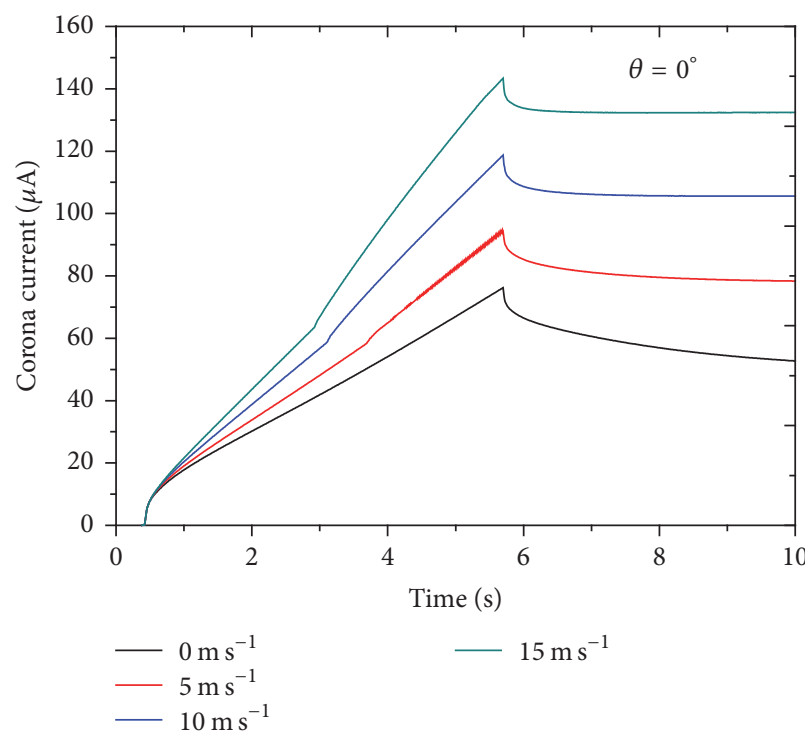

(a)

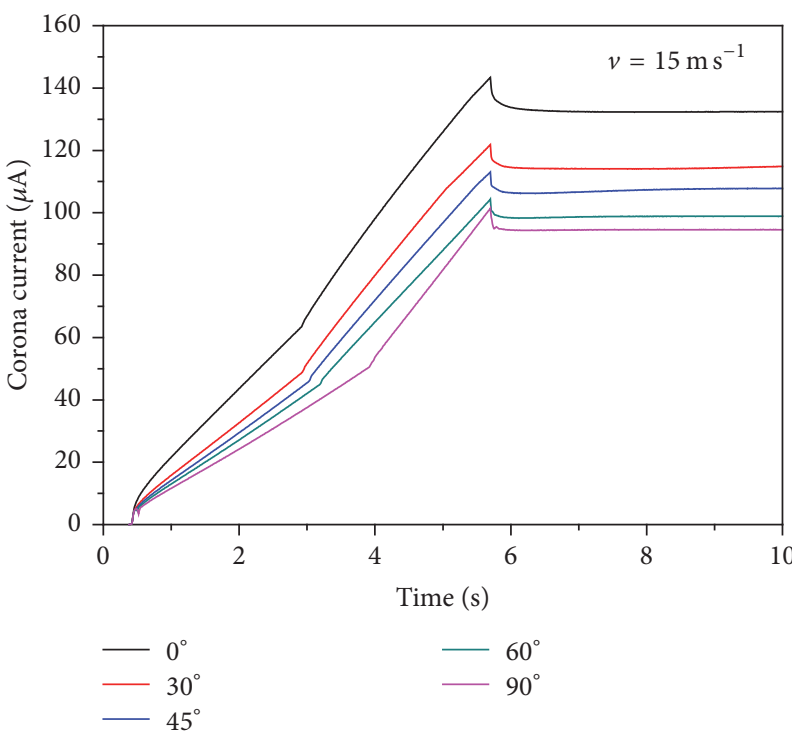

(b)

FIGURE 7: The time-evolution of the corona current for the same blowing directions but with different wind speeds (a) and for the same wind speeds but with different blowing directions (b).

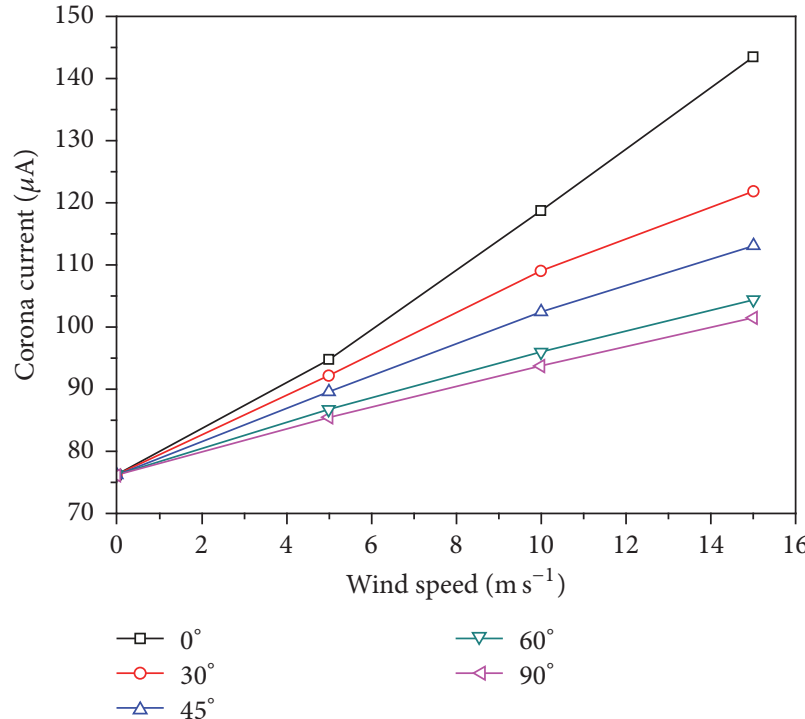

(a)

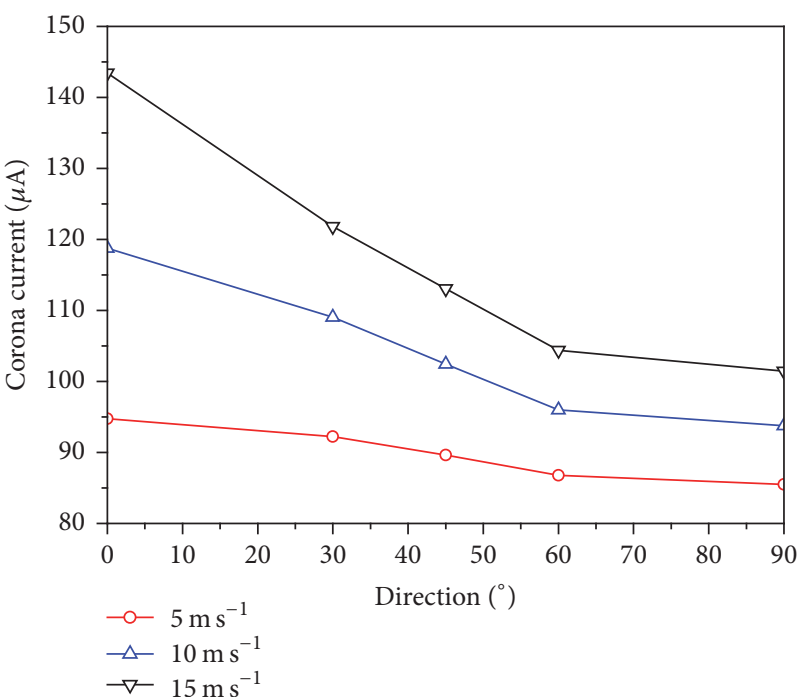

(b)

FIGURE 8: The peak corona current as a function of the velocity (a) and the direction (b) of wind.

component is much larger than the vertical component, the larger wind speed will result in a higher field value. When the horizontal wind component is less than the vertical wind component, the larger wind speed may result in a lower field value. Because the larger horizontal wind speed results in a more obvious drift of the corona ions, which further reduces the screening effect on the local field of point $\mathrm{A}$. The larger vertical wind speed leads to an accumulation of corona ions upon the rod tip, which further increase the screening effect on the local field of point A. Thirdly, it was shown that the more horizontal components or the larger speed will result in a larger corona current, because both of them can cause the positive corona ions to drift away from the rod tip. However, when the wind speed is less than $5 \mathrm{~m} \mathrm{~s}^{-1}$, the effect of wind direction on the corona current is not obvious.

Furthermore, the results of this study support the reasons explained by Wang and Takagi [8] why the upward lightning self-initiated on a tall object is commonly observed under a strong wind condition and the electric field observed at ground level is smaller than normal. Because only when the strong wind is with a large horizontal components and a small vertical components, the shielding effects of the corona space 
charges on the object tip are small. But in the meantime it will cause a large shielding effect on the leeside and make the electric field at ground level small. Based on the result of this study and the observation of Wang and Takagi [8], it can be supposed that the upward lightning or leaders might be initiated easier from the objects when a strong wind with large horizontal components and small vertical components is blowing. Thus, it should be noted that the direction of the wind as a parameter is important as the wind speed, no matter whether in numerical simulations or in observation experiments, when studying the effect of wind on corona discharges and on lightning initiations and attachments. In addition, the results of our numerical simulation are potentially important to those who are studying not only the behaviors of upward leader initiations and propagation, but also the lightning protections. But we remind the reader that the uncertainties in the technique used herein to calculate the electric field and estimate the corona charge distribution by a two-dimensional numerical model may yield some errors. We will be dedicated to developing the 3D model with fine mesh in the future, for achieving a more accurate result and for quantitative analysis. Direct measurements of the charge density around the tip of objects and the corona current in those tips under thunderstorm condition at different wind conditions, especially at different wind directions, are needed to verify our tentative findings. Furthermore, only one kind of structure is considered for studying the effect of wind on corona discharge. What about the corona discharge on other structures affected by wind, such as on a building with a flat roof, a windmill, or a skyscraper? And it will be our future work.

\section{Conflicts of Interest}

The authors declare that there are no conflicts of interest regarding the publication of this paper.

\section{Acknowledgments}

This work was supported in part by National Natural Science Foundation of China under Grants 41575004 and 41275009, in part by the National Key Basic Research Program of China (2014CB441405), in part by the Power Grid Lightning Warning Technology Research and the Lightning Warning System Development (K-YN2013-186), and in part by Distribution Network Study and Engineering Demonstration of Integrated Lightning Protection System (YNKJQQ00000274) and by the project funded by the Priority Academic Program Development of Jiangsu Higher Education Institutions (PAPD: KYZZ-0243).

\section{References}

[1] D. R. Macgorman, W. D. Rust, and E. R. Williams, "The electrical nature of storms," Physics Today, vol. 52, no. 52, pp. 68-70, 2008.

[2] S. Chauzy and P. Raizonville, "Space charge layers created by coronae at ground level below thunderclouds: Measurements and modeling," Journal of Geophysical Research, vol. 87, no. C4, p. 3143, 1982.

[3] S. Chauzy and S. Soula, "General interpretation of surface electric field variations between lightning flashes," Journal of Geophysical Research, vol. 92, no. D5, p. 5676, 1987.

[4] S. Chauzy, J. Médale, S. Prieur, and S. Soula, "Multilevel measurement of the electric field underneath a thundercloud: 1. A new system and the associated data processing," Journal of Geophysical Research, vol. 96, no. D12, p. 22319, 1991.

[5] S. Soula, "Transfer of electrical space charge from corona between ground and thundercloud: Measurements and modeling," Journal of Geophysical Research, vol. 99, no. D5, p. 10759, 1994.

[6] X. Qie, S. Soula, and S. Chauzy, "Influence of ion attachment on the vertical distribution of the electric field and charge density below a thunderstorm," Annales Geophysicae, vol. 12, no. 12, pp. 1218-1228, 1994.

[7] R. H. Golde and R. Golde, Lightning: Physics of Lightning, vol. 2, Academic Press, 1977.

[8] D. Wang and N. Takagi, "Characteristics of Winter Lightning that Occurred on a Windmill and its Lightning Protection Tower," IEEJ Transactions on Power and Energy, vol. 131, no. 7, pp. 532-535, 2011.

[9] N. L. Aleksandrov, E. M. Bazelyan, and Y. P. Raizer, "Initiation and development of first lightning leader: The effects of coronae and position of lightning origin," Atmospheric Research, vol. 76, no. 1-4, pp. 307-329, 2005.

[10] E. M. Bazelyan, Y. P. Raizer, and N. L. Aleksandrov, "Corona initiated from grounded objects under thunderstorm conditions and its influence on lightning attachment," Plasma Sources Science and Technology, vol. 17, no. 2, Article ID 024015, 2008.

[11] J. A. Chalmers, Atmospheric Electricity, Atmospheric Electricity, 2nd edition, 1967.

[12] F. D'Alessandro, "Experimental study of the effect of wind on positive and negative corona from a sharp point in a thunderstorm," Journal of Electrostatics, vol. 67, no. 2-3, pp. 482487, 2009.

[13] E. M. Bazelyan, Y. P. Raizer, N. L. Aleksandrov, and F. D'Alessandro, "Corona processes and lightning attachment: The effect of wind during thunderstorms," Atmospheric Research, vol. 94, no. 3, pp. 436-447, 2009.

[14] B. X. Du, H. Xu, and Y. Liu, "Effects of wind condition on hydrophobicity behavior of silicone rubber in corona discharge environment," IEEE Transactions on Dielectrics and Electrical Insulation, vol. 23, no. 1, pp. 385-393, 2016.

[15] Y. Zou, J. He, and H. He, "Effect of wind on the distribution of the corona discharge ionized field generated from grounded wire during thunderstorms," in Proceedings of the 2014 International Conference on Lightning Protection, ICLP 2014, pp. 879884, chn, October 2014.

[16] N. L. Aleksandrov, E. M. Bazelyan, M. M. Drabkin, R. B. Carpenter Jr., and Y. P. Raizer, "Corona discharge at the tip of a tall object in the electric field of a thundercloud," Plasma Physics Reports, vol. 28, no. 11, pp. 953-964, 2002.

[17] M. Becerra, "Glow corona generation and streamer inception at the tip of grounded objects during thunderstorms: Revisited," Journal of Physics D: Applied Physics, vol. 46, no. 13, Article ID 135205, 2013.

[18] X. Guo and Q. Zhang, "Effects of geometrical parameters of two height-unequal adjacent objects on corona discharges from their tips during a thunderstorm," Atmospheric Research, vol. 190, pp. 113-120, 2017. 
[19] R. Waters, T. Rickard, and W. Stark, "Direct measurement of electric field at line conductors during a.c. corona," Proceedings of the Institution of Electrical Engineers, vol. 119, no. 6, pp. 717723, 1972.

[20] L. Liu and M. Becerra, "On the transition from stable positive glow corona to streamers," Journal of Physics D: Applied Physics, vol. 49, no. 22, Article ID 225202, 2016.

[21] M. Stolzenburg, W. D. Rust, and T. C. Marshall, "Electrical structure in thunderstorm convective regions 2. Isolated storms," Journal of Geophysical Research Atmospheres, vol. 103, no. 12, Article ID 97JD03547, pp. 14079-14096, 1998.

[22] Y. Tan, X. Guo, J. Zhu, Z. Shi, and D. Zhang, "Influence on simulation accuracy of atmospheric electric field around a building by space resolution," Atmospheric Research, vol. 138, pp. 301-307, 2014.

[23] D. Ariza, F. Roman, O. Escobar, and O. Montero, "Corona current and space charge during a thunderstorm," in Proceedings of the 2012 31st International Conference on Lightning Protection, ICLP 2012, aut, September 2012.

[24] G. J. Byrne, A. A. Few, and M. F. Stewart, "The effects of atmospheric parameters on a corona probe used in measuring thunderstorm electric fields," Journal of Geophysical Research, vol. 91, no. D9, p. 9911, 1986.

[25] S. Sakata and T. Okada, "Effect of humidity on hydrated clusterion formation in a clean room corona discharge neutralizer," Journal of Aerosol Science, vol. 25, no. 5, pp. 879-893, 1994.

[26] Q. Hu, L. Shu, X. Jiang, C. Sun, Z. Qiu, and R. Lin, "Influence of air pressure and humidity on positive direct current corona discharge performances of the conductor in a corona cage," International Transactions on Electrical Energy Systems, vol. 24, no. 5, pp. 723-735, 2014. 


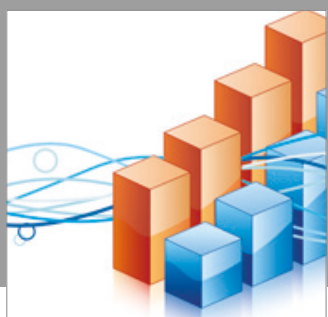

Advances in

Operations Research

vatersals

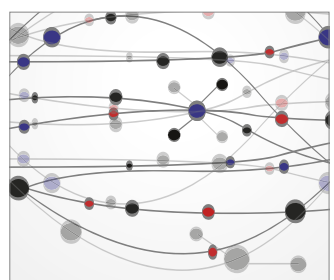

\section{The Scientific} World Journal
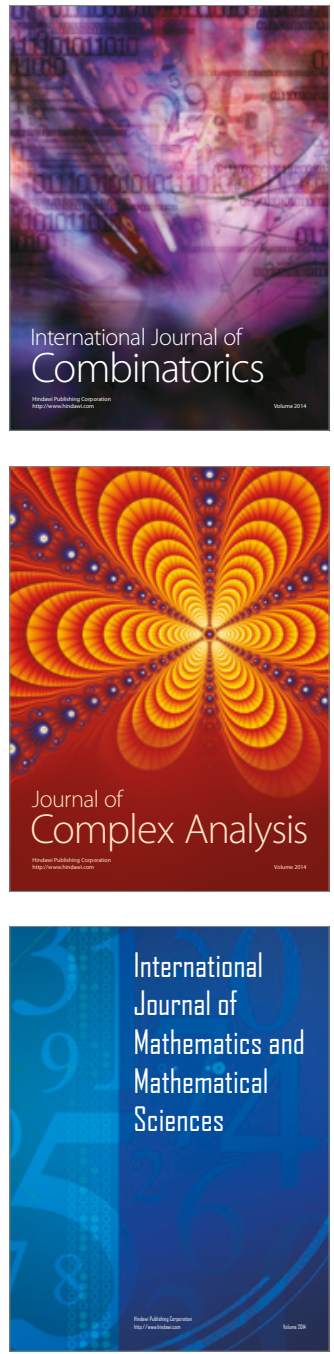
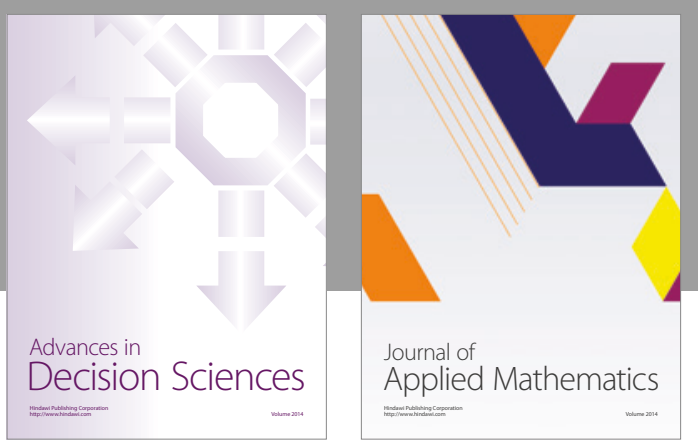

Algebra

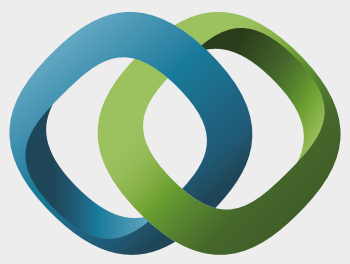

\section{Hindawi}

Submit your manuscripts at

https://www.hindawi.com
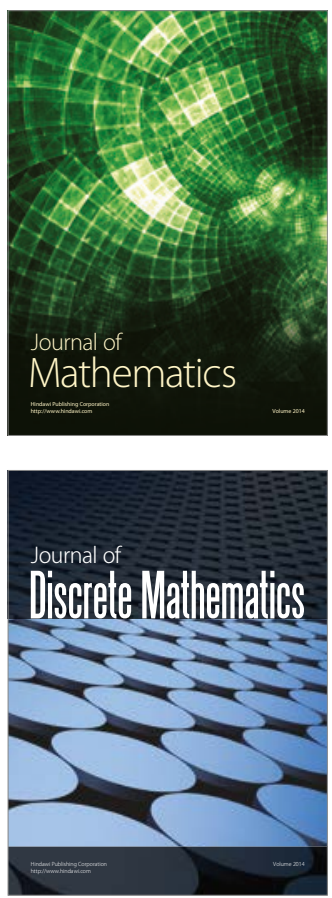

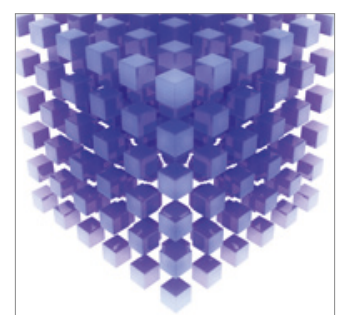

Mathematical Problems in Engineering
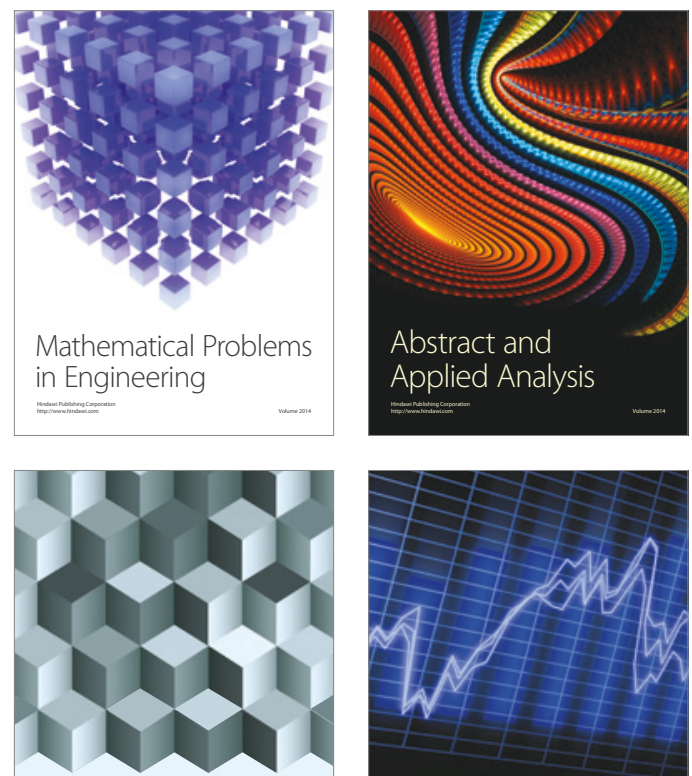

Journal of

Function Spaces

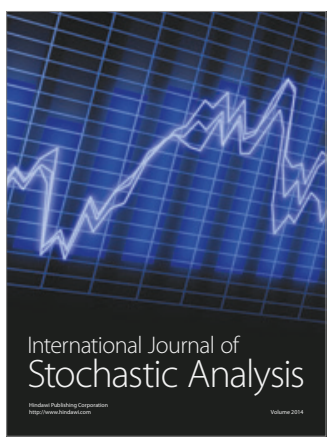

Probability and Statistics
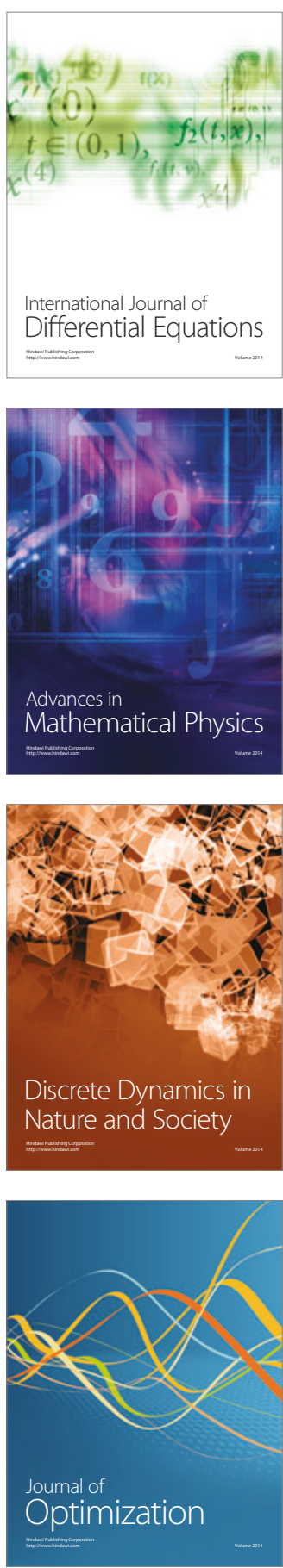